\title{
Anesthetic management of one-stage scheduled surgery for adrenal cortical carcinoma complicated by massive pulmonary tumor embolism
}

\author{
Kazuma Yunoki ${ }^{1 *}$ D, Tsutomu Wada $^{2}$, Ikuko Miyawaki ${ }^{1}$, Kazuo Yamazaki ${ }^{1}$ and Hiroyuki Mima ${ }^{1}$
}

Keywords: Adrenal cortical carcinoma, Cardiopulmonary bypass, Inferior vena cava, One-stage surgery, Pulmonary tumor embolism, Transesophageal echocardiography

\section{Background}

Adrenal cortical carcinoma (ACC) with pulmonary tumor embolism (PTE) occurs extremely infrequently, and no case reports are available concerning one-stage scheduled resection of ACC and PTE with cardiopulmonary bypass $(\mathrm{CPB})$. Herein, we report a successful management of patient with ACC complicated by a massive PTE.

\section{Case presentation}

A 44-year-old, $150.0 \mathrm{~cm}, 85.0 \mathrm{~kg}$ female was transferred to our hospital complaining of dyspnea. T1-weighted magnetic resonance imaging revealed a large left adrenal tumor extending into the IVC with its proximal position at the level of the hepatic vein (Fig. 1), and contrast-enhanced CT revealed a massive PTE occluding the right pulmonary artery (PA) (Fig. 2). Transthoracic echocardiography (TTE) revealed moderate tricuspid regurgitation (TR) and a diastolic D-shaped left ventricle. IVC filter placement was not performed due to technical difficulties. Laboratory test results revealed elevated levels of serum cortisol and reduced levels of serum adrenocorticotropic hormone. These findings were suggestive of an ACC. Surgical treatment was considered necessary for the prevention of a lethal PTE, and one-stage surgery was scheduled. This procedure included a left nephrectomy, resection of the IVC tumor thrombus, and removal of the PTE under CPB.

\footnotetext{
* Correspondence: yunokik@kcho.jp

${ }^{1}$ The Department of Anesthesia and Critical Care, Kobe City Medical Center General Hospital, 2-1-1, Minatojimaminamimachi, Chuo-ku, Kobe city, Hyogo 6500047, Japan

Full list of author information is available at the end of the article
}

General anesthesia was induced with Propofol, rocuronium, and fentanyl and was followed by endotracheal intubation. After the placement of a transesophageal echocardiography (TEE) probe (Fig. 3) and left radial arterial catheter, a pulmonary artery catheter (PAC) were inserted from the right internal jugular vein and placed with its tip temporarily in the right atrium. A median sternotomy was initially performed for the preparation of an emergent $\mathrm{CPB}$ and was followed by abdominal incision. After IVC clamping at the proximal level of the abdominal IVC, the tumor mass was removed coupled with the left adrenal tumor, left renal vein, and IVC tumor thrombus. The hemostasis procedure was a bit challenging due to the bleeding from the swollen abdominal vessels surrounding the tumor. In total, $2800 \mathrm{~mL}$ of blood was lost during the abdominal procedure.

After the confirmation that abdominal hemostasis had been complete, PTE resection started. After systemic heparinization, followed by cannulation of the ascending aorta, a total flow bypass with superior vena cava (SVC) and IVC cannulation was established. Significant abdominal bleeding was not observed. PA tumor embolectomy was completed under cardiac arrest, and the tip of the PAC was manually guided into the main PA. The patient was successfully weaned from the $\mathrm{CPB}$. The total aortic cross-clamping and CPB times were 38 and $97 \mathrm{~min}$, respectively. Pulmonary arterial pressure (PAP) was maintained within normal limits. The total duration of the operation was $13 \mathrm{~h}$ and $55 \mathrm{~min}$. The total amount of bleeding was $4900 \mathrm{~mL}$, and $1960 \mathrm{~mL}$ of red blood cell products were infused. The patient was extubated on the 


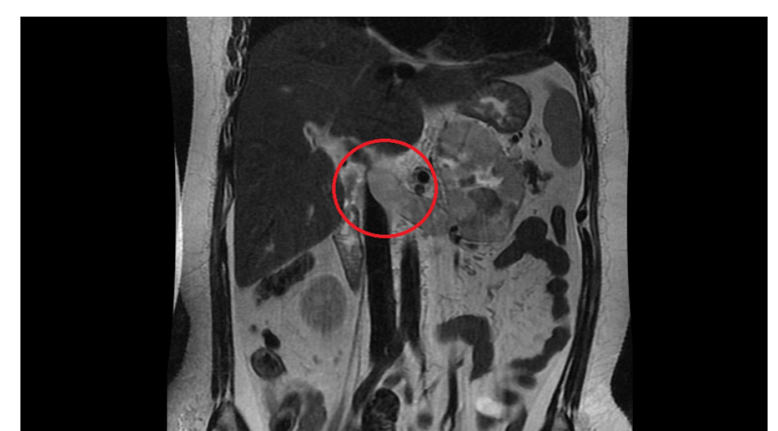

Fig. 1 Abdominal magnetic resonance imaging. T1-weighted magnetic resonance image revealing a large $(10.0 \mathrm{~cm})$ tumor in the left adrenal gland extending into the inferior vena cava (circle) with the proximal position at the level of the hepatic vein

next day and was discharged from hospital on postoperative day 16, without any complications.

\section{Discussion}

One in 10 patients with renal cell carcinoma (RCC) are reported to present with IVC tumor invasion. Of these, $1.0 \%$ present with right atrial invasion $[1,2]$. In comparison, ACC is a relatively rare cancer and ACC with IVC extension occurs extremely infrequently. Surgical resection is the first choice of treatment if complete removal of the tumor is expected [3]. In our case, though radical surgical removal of the tumor was considered infeasible, surgical treatment was selected to prevent lethal PTEs.

The initial concern regarding this procedure is hemorrhaging. IVC obstructive tumors tend to develop collateral veins around the tumor, and surgical hemostasis is often challenging. Complete hemostasis is, however, required in the abdominal procedure, because the patient is to be heparinized for $\mathrm{CPB}$. If abdominal hemostasis is incomplete, we must consider postponing the pulmonary embolectomy. Furthermore, performing $\mathrm{CPB}$ in as short a time as

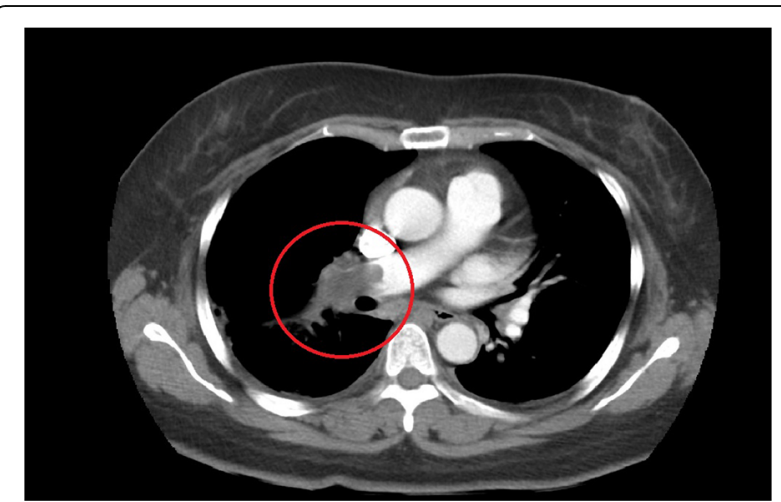

Fig. 2 Thoracic contrast-enhanced computed tomography. Contrastenhanced computed tomography scan revealing a massive pulmonary tumor embolism (circle) occluding the right pulmonary artery

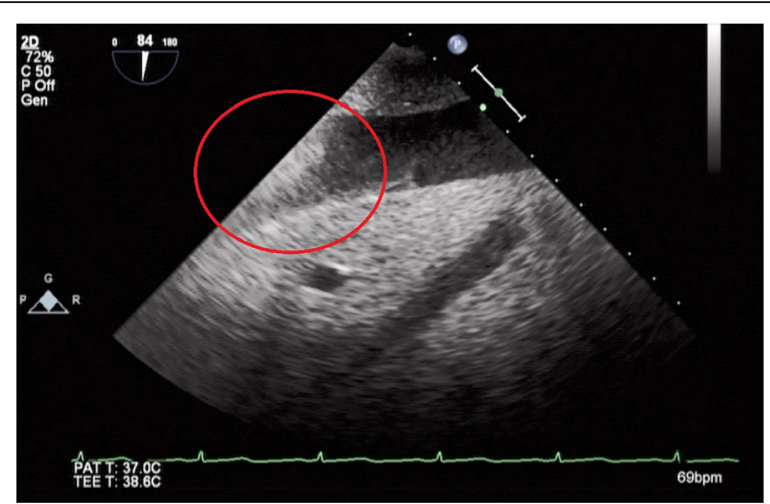

Fig. 3 Intraoperative transthoracic echocardiography. Intraoperative transthoracic echocardiography image of the modified transgastric hepatic vein view revealing that the inferior vena cava was almost fully occluded (circle) by a tumor embolus at the level of the inferior hepatic vein

possible and preventing hypothermia may contribute to the preservation of platelet function and the coagulation system.

The second concern is the unanticipated formation of new PTEs. While no reports have been published concerning the risk of PTEs in ACC patients, this case suggests ACC with IVC invasion can cause massive PTESs. So, we should be prepared for abrupt migration of tumor in the case of ACC. The importance of TEE for IVC invading tumors is well recognized [4], and TEE monitoring is recommended for stages 3 and 4 patients [5]. Our patient was classified as stage 2 disease, and TEE monitoring is usually not recommended. Given that the risk of intraoperative tumor migration is still unknown in the case of ACC, however, TEE observation should be necessary even though this is classified as stage 2 in the case of ACC.

\section{Conclusion}

One-stage scheduled surgery for resection of ACC and PTE under CPB was performed successfully. Intraoperative TEE is a useful monitor since the risk of acute PTE by migration of the tumor has yet to be determined in the case of ACC.

\section{Abbreviations \\ ACC: Adrenal cortical carcinoma; CPB: Cardiopulmonary bypass: \\ CT: Computed tomography; ICU: Intensive care unit; IVC: Inferior vena cava; PA: Pulmonary artery; PAC: Pulmonary artery catheter; PAP: Pulmonary arterial pressure; PTE: Pulmonary thromboembolism; PV: Pulmonary vein; RCC: Renal cell carcinoma; SVC: Superior vena cava; TEE: Transesophageal echocardiography; TR: Tricuspid regurgitation; TTE: Transthoracic echocardiography}

\section{Author details}

'The Department of Anesthesia and Critical Care, Kobe City Medical Center General Hospital, 2-1-1, Minatojimaminamimachi, Chuo-ku, Kobe city, Hyogo 6500047, Japan. ${ }^{2}$ The Department of Anesthesiology, Rinku General Medical Center, Rinku Ourai Kita 2-23, Izumisano city, Osaka 5988577, Japan. 
Received: 24 July 2017 Accepted: 16 August 2017

Published online: 02 September 2017

\section{Authors' contributions}

KY1 wrote the case report. KY1 and TW conducted anesthetic management to the patient. IM and KY2 helped to draft the manuscript. HM conducted

the ICU treatments. All authors read and approved the final manuscript.

\section{Consent for publication}

Written informed consent was obtained from the patient for the publication of this case report and any accompanying images.

\section{Competing interests}

The authors declare that they have no competing interests.

\section{Publisher's Note}

Springer Nature remains neutral with regard to jurisdictional claims in published maps and institutional affiliations.

\section{Author details}

${ }^{1}$ The Department of Anesthesia and Critical Care, Kobe City Medical Center General Hospital, 2-1-1, Minatojimaminamimachi, Chuo-ku, Kobe city, Hyogo 6500047, Japan. ${ }^{2}$ The Department of Anesthesiology, Rinku General Medical Center, Rinku Ourai Kita 2-23, Izumisano city, Osaka 5988577, Japan.

Received: 24 July 2017 Accepted: 16 August 2017

Published online: 02 September 2017

\section{References}

1. Ciancio G, Manoharan M, Katkoori D, et al. Long-term survival in patients undergoing radical nephrectomy and inferior vena cava thrombectomy: single-center experience. Eur Urol. 2010;57:667-72.

2. Marshall FF, Dietrick DD, Baumgartner WA, et al. Surgical management of renal cell carcinoma with intracaval neoplastic extension above the hepatic veins. J Urol. 1988;139:1166-72.

3. Laan DV, Thiels CA, Glasgow A, et al. Adrenocortical carcinoma with inferior vena cava tumor thrombus. Surgery. 2017;161:240-8.

4. Fukazawa K, Gologorsky E, Naguit K, et al. Invasive renal cell carcinoma with inferior vena cava tumor thrombus: cardiac anesthesia in liver transplant settings. J Cardiothorac Vasc Anesth. 2014;28:640-6.

5. Neves RJ, Zincke H. Surgical treatment of renal cancer with vena cava extension. Br J Urol. 1987:59:390-5.

\section{Submit your manuscript to a SpringerOpen ${ }^{\circ}$ journal and benefit from:}

- Convenient online submission

- Rigorous peer review

- Open access: articles freely available online

- High visibility within the field

- Retaining the copyright to your article 